\title{
Optically controlled resonance energy transfer: Mechanism and configuration for all-optical switching
}

\author{
David S. Bradshaw and David L. Andrews ${ }^{\text {a) }}$ \\ Nanostructures and Photomolecular Systems, School of Chemical Sciences, University of East Anglia, \\ Norwich NR4 7TJ, United Kingdom
}

(Received 14 January 2008; accepted 13 February 2008; published online 10 April 2008)

\begin{abstract}
In a molecular system of energy donors and acceptors, resonance energy transfer is the primary mechanism by means of which electronic energy is redistributed between molecules, following the excitation of a donor. Given a suitable geometric configuration it is possible to completely inhibit this energy transfer in such a way that it can only be activated by application of an off-resonant laser beam: this is the principle of optically controlled resonance energy transfer, the basis for an all-optical switch. This paper begins with an investigation of optically controlled energy transfer between a single donor and acceptor molecule, identifying the symmetry and structural constraints and analyzing in detail the dependence on molecular energy level positioning. Spatially correlated donor and acceptor arrays with linear, square, and hexagonally structured arrangements are then assessed as potential configurations for all-optical switching. Built on quantum electrodynamical principles the concept of transfer fidelity, a parameter quantifying the efficiency of energy transportation, is introduced and defined. Results are explored by employing numerical simulations and graphical analysis. Finally, a discussion focuses on the advantages of such energy transfer based processes over all-optical switching of other proposed forms. (C) 2008 American Institute of Physics. [DOI: $10.1063 / 1.2894319]$
\end{abstract}

\section{INTRODUCTION}

As the speed of ultrafast communications, sensing, and computer processing maintains an apparently unabated rise, the need to achieve ever faster switching capability becomes increasingly pressing. Although the pace of such improvement in electronic devices still continues more or less according to Moore's Law, all-optical systems have a clear potential for much greater bandwidths and speedscircumventing the bottlenecks that can result from optoelectronic conversion. For many years it has been known that all-optical switching, based on various forms of photonic interaction in which light is controlled by light, is not only technically realizable but has the capacity to revolutionize telecommunications and computing. Not surprisingly, numerous implementation strategies have been entertained, and many are the subject of vigorous ongoing research.

In recent attempts to deliver a robust basis for this eagerly anticipated technology, numerous fundamentally different mechanisms and materials have been proposed. Many efforts to deploy silicon as the basis for optical switching systems and devices have proved problematic due to the relatively weak nonlinear optical properties of the material, although some successes have been reported. ${ }^{1,2}$ A great deal of research activity now centers on semiconductor quantum well systems ${ }^{3-10}$ that offer opportunities to exploit the inter subband excitation transitions of confined electrons, associated with ultrafast relaxation. Based on similar principles, the feasibility of using carbon nanotubes, of morphology appropriate for all-optical switching, has also been

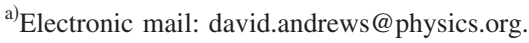

demonstrated. ${ }^{11,12}$ In most semiconductor devices optical switching is conceived as an exploitation of optical saturation - though an alternative method, based on quantum interference in electromagnetic induced transparency (EIT) ${ }^{13-15}$ has recently been demonstrated in rubidium atoms. ${ }^{16-18}$ Furthermore, in a novel development by Dawes et $a l .{ }^{19}$ (based on the same medium), counterpropagating laser beams have been shown to induce an optical pattern that rotates on application of a switching laser.

Elsewhere there is also continued interest in optical switching schemes based on classical mechanisms for interferometric switching, in either Mach-Zehnder ${ }^{20-24}$ or Sagnac $^{25,26}$ configurations, engaging optical phase shifts to provide constructive or destructive interference-many of these (and other) processes are detailed in a recent review by Wada. ${ }^{27}$ Beyond the more widely discussed methods, a number of other concepts repeatedly resurface in the primary literature. These include, for example, systems based on photonic crystals with cross-waveguide geometries, offering switching action through the optical Kerr effect; ${ }^{28-30}$ surfaceplasmon polariton media, in which light-induced dielectric modification at an interface affects the transmission of throughput radiation; ${ }^{31-33}$ azobenzene-containing materials, which have differing optical properties for the trans and cis configurations, reversibly interchangeable by photoisomerization, ${ }^{34-36}$ and various schemes based on films of bacteriorhodopsin, a light-harvesting protein with unique nonlinear optical properties. ${ }^{37-41}$

The present paper reports an in-depth analysis of a very different all-optical switching mechanism-initially outlined by one of the present authors ${ }^{4-44}$-based on the optical con- 


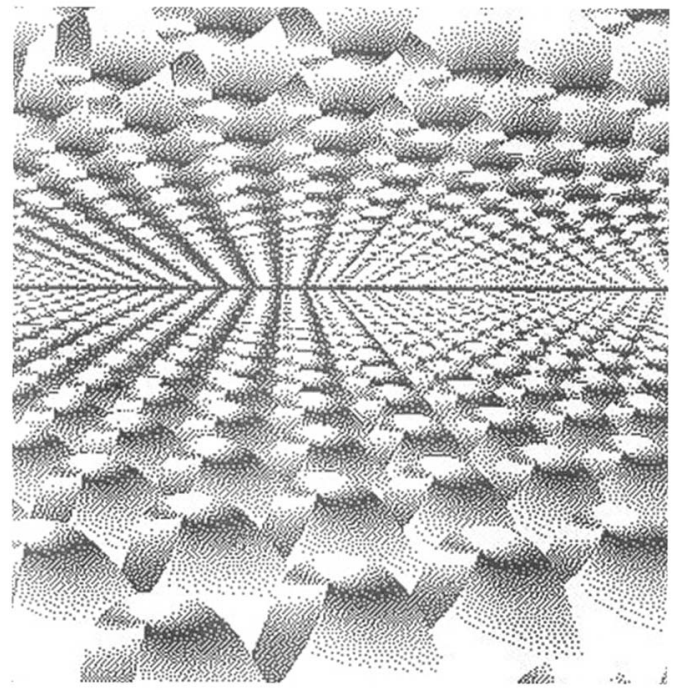

FIG. 1. Artistic impression of parallel two-dimensional donor and acceptor arrays, each arranged in the form of a hexagonal lattice.

trol of resonance energy transfer (RET) between molecules. When RET occurs spontaneously, following the absorption of light, it usually represents the principal process for the intermolecular translation of electronic energy, from the sites of initial optical excitation. ${ }^{45}$ However, under suitable conditions such processes of energy transfer can be activated or deactivated by (nonresonant) optical stimulation. In many features, optical switches based on this principle differ strikingly from the majority of previously proposed schemes. Here, photonic switching is localized in molecular pairs rather than ensembles, and the mechanism allows much shorter operating wavelengths to be engaged, i.e., the ultraviolet or visible range, in contrast to the infrared. These features offer significant promise for miniaturized array implementation, illustrated pictorially by Fig. 1.

In its simplest form known as Förster transfer, RET involves a relocation of energy from an electronically excited donor $A$ to an acceptor $B$ in its ground state. By input of an auxiliary laser field this energy transfer may be enhanced by a laser-assisted RET (LARET) mechanism, whose efficiency scales linearly with the laser intensity. ${ }^{46}$ Calculations have shown that LARET can offer a several-fold increase in the rate of energy transfer, even for modest pulsed laser irradiances of around $10^{12} \mathrm{~W} \mathrm{~cm}^{-2}$. ${ }^{47}$ Optically controlled RET (OCRET), the mechanistic basis for the study reported here, is a related process, differing from LARET in that an important configurable condition is applied. This condition requires the transition dipole moments of the donor and acceptor, and their mutual displacement vector, to be mutually perpendicular - thus excluding a Förster process that would otherwise be possible on symmetry and energetic grounds. ${ }^{48}$ By application of the off-resonant laser beam the transfer of energy is activated, effecting all-optical switching action.

The following analysis begins by developing the fundamental electrodynamic theory for the OCRET mechanism, identifying in detail the geometric, optical, spectroscopic, and molecular structural conditions for it to occur between a single donor-acceptor pair (Sec. II). The analysis is then ex- tended to several arrangements that could be useful for realistic all-optical switches, taking into account the requirement for a multiplicity of such donor-acceptor partners to operate independently, without significant cross-talk. The specific geometric configurations to be examined are onedimensional linear-arrays (Sec. III), two-dimensional squarelattice arrays (Sec. IV), and hexagonal-lattice arrays (Sec. V). Further implementation issues are addressed in the concluding Sec. VI, focusing on the advantages of OCRET over other forms of all-optical switch.

\section{COUPLING PAIR}

The theory of choice for systems involving lightmolecule interactions is nonrelativistic quantum electrodynamics (QED) - the basis for not only the present analysis, but also another recently proposed all-optical switch based on EIT, associated with a different mechanism. ${ }^{15}$ In earlier work, both RET and LARET have been fully analyzed using $\mathrm{QED}$, research that has laid the foundation for this paper. To specifically determine the rate of energy transfer for the OCRET mechanism, a time-dependent perturbation theory method is required. Details of the calculation, which is used to establish the quantum amplitudes of the system, are fully described in Refs. 46 and 47 and they are not repeated here. The OCRET mechanism is distinguished by the fact that the quantum amplitude for Förster transfer has a null result-a consequence of the orthogonal triad condition, i.e., $\mathbf{R} \perp \boldsymbol{\mu}_{A} \perp \boldsymbol{\mu}_{B}$, where $\mathbf{R}$ is the donor-acceptor displacement vector $\overrightarrow{A B}$, and $\boldsymbol{\mu}_{A}, \boldsymbol{\mu}_{B}$ are the salient transition dipole moments of the two molecules. ${ }^{49}$ In the near-field, the quantum amplitude arising from the input auxiliary beam is given by

$$
\begin{aligned}
M_{f i}^{(4)}= & \left(\frac{n \hbar c k}{8 \pi \varepsilon_{0}^{2} V R^{3}}\right) e_{i} \bar{e}_{l}\left(\delta_{j k}-3 \hat{R}_{j} \hat{R}_{k}\right)\left(S_{i j}^{A}(k) S_{l k}^{B}(-k)\right. \\
& \left.+S_{i j}^{B}(k) S_{l k}^{A}(-k)+T_{i j l}^{A}(k) \mu_{k}^{B}+T_{i j l}^{B}(k) \mu_{k}^{A}\right),
\end{aligned}
$$

where $n$ is the number of photons (proportional to irradiance) in the quantization volume $V$, and the implied summation convention for repeated Cartesian tensor indices is employed; $\mu_{k}^{\xi}$ is a static (permanent) dipole moment and, as defined below, $S_{i j}^{\xi}( \pm k)$ and $T_{i j k}^{\xi}(k)$ are the generalized polarizability and hyperpolarizability tensors, respectively. Furthermore, $\mathbf{e}$ and $\hbar c k$ denote the polarization vector (an overbar denoting complex conjugation) and energy of the input photon, respectively. Each of the four terms of Eq. (2.1), illustrated by the Feynman diagrams of Figs. 2(a)-2(d), represents a fourth-order photonic interaction event, necessitating deployment of a fourth-order perturbation treatment as indicated by the superscript of $M_{f i}$. An energy scheme representing this mechanism is illustrated in Fig. 3.

Returning to the optical response tensors in Eq. (2.1), these are explicitly given by the following:

$$
S_{i j}^{\xi}( \pm k)=\sum_{r}\left\{\frac{\mu_{i}^{f r} \mu_{j}^{r i}}{\widetilde{E}_{r f} \pm \hbar c k}+\frac{\mu_{j}^{f r} \mu_{i}^{r i}}{\widetilde{E}_{r i} \mp \hbar c k}\right\}
$$




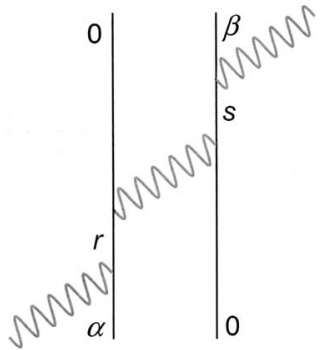

(a)

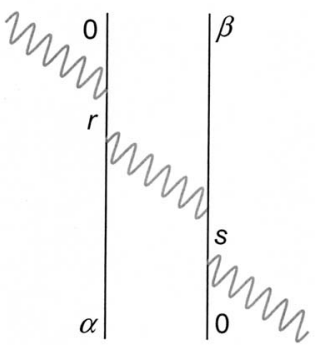

(b)

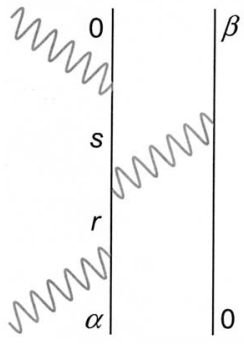

(c)

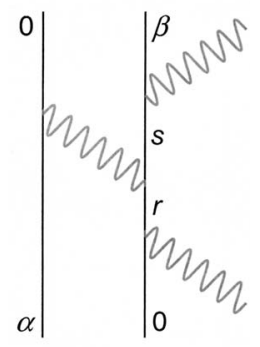

(d)
FIG. 2. Four Feynman diagrams for OCRET. Here, $|0\rangle$ represents a molecule in the ground state; $|\alpha\rangle$ and $|\beta\rangle$ relate to the excited state of the donor (on the left) and acceptor (right), respectively, with $|r\rangle$ and $|s\rangle$ as the corresponding intermediate states. In detail, diagram (a) depicts an instantaneous mechanism involving photon absorption and emission at the donor and acceptor, respectively, with a coupling photon created at the donor and annihilated at the acceptor; thus excitation is transferred from $A$ to $B$. Diagrams (b), (c), and (d) are permutations that will achieve an identical final result.

$$
\begin{aligned}
T_{i j l}^{\xi}(k)= & \sum_{r, s}\left\{\frac{\mu_{i}^{f s} \mu_{j}^{s r} \mu_{l}^{r i}}{\left(\widetilde{E}_{r i}+\hbar c k\right)\left(\widetilde{E}_{s f}+\hbar c k\right)}+\frac{\mu_{i}^{f s} \mu_{l}^{s r} \mu_{j}^{r i}}{\widetilde{E}_{r f}\left(\widetilde{E}_{s f}+\hbar c k\right)}\right. \\
& +\frac{\mu_{j}^{f s} \mu_{i}^{s r} \mu_{l}^{r i}}{\left(\widetilde{E}_{r i}+\hbar c k\right) \widetilde{E}_{s i}}+\frac{\mu_{j}^{f s} \mu_{l}^{s r} \mu_{i}^{r i}}{\left(\widetilde{E}_{r i}-\hbar c k\right) \widetilde{E}_{s i}} \\
& \left.+\frac{\mu_{l}^{f s} \mu_{i}^{s r} \mu_{j}^{r i}}{\widetilde{E}_{r f}\left(\widetilde{E}_{s f}-\hbar c k\right)}+\frac{\mu_{l}^{f s} \mu_{j}^{s r} \mu_{i}^{r i}}{\left(\widetilde{E}_{r i}-\hbar c k\right)\left(\widetilde{E}_{s f}-\hbar c k\right)}\right\},
\end{aligned}
$$

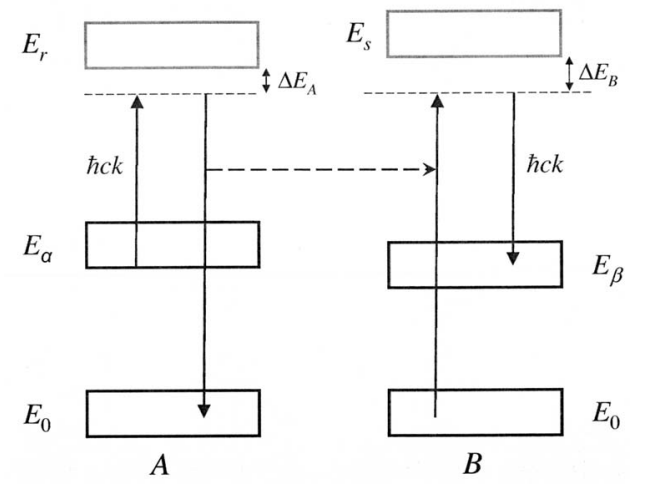

FIG. 3. Energy scheme for OCRET from $A$ to $B$. Here, vertical arrows denote four interactions coupling the donor decay $|\alpha\rangle \rightarrow|0\rangle$ to the acceptor excitation $|0\rangle \rightarrow|\beta\rangle$. The directly involved energy levels are: $E_{0}$, representing the ground electronic state for each molecule, and; $E_{\alpha}$ and $E_{\beta}$, the electronic excited states of $A$ and $B$, respectively. Each state has its own vibrational manifold. Dashed lines denote virtual states, the closest energy levels $E_{r}$ and $E_{s}$ (not directly involved in the process, depicted in gray) being offset in energy by $\Delta E_{A}$ and $\Delta E_{B}$. The horizontal arrow signifies energy transfer.

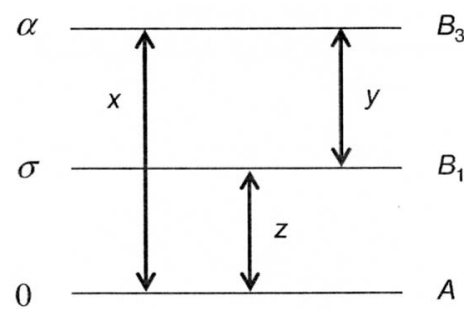

(a)

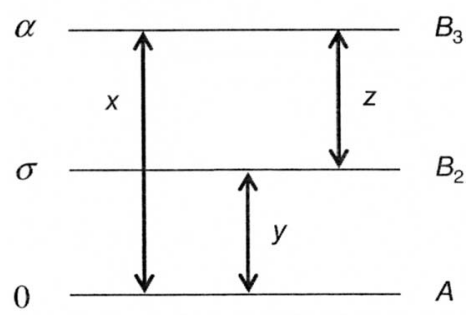

(b)

FIG. 4. Energy level positionings of states $|0\rangle,|\sigma\rangle$, and $|\alpha\rangle$ for donor $A$ and their corresponding symmetry classes for point group symmetry $D_{2}$. Both possible classes of intermediate state, i.e., $B_{1}$ or $B_{2}$, are shown, and the transformation properties for each allowed transition are indicated.

respectively, where the transition dipole moments are designated by the shorthand notation $\boldsymbol{\mu}^{x y}=\langle x|\boldsymbol{\mu}| y\rangle$, energy differences $E_{x y}=E_{x}-E_{y}$, and tildes denote the necessity of including damping terms if the theory were to be developed for near-resonance conditions ${ }^{50-56}$ - however, the present analysis will solely focus on off-resonant photon absorption, and henceforth the tildes can be omitted. It is important to note that the transition dipoles entailed in Eqs. (2.2) and (2.3) represent complete sets, not limited to $\hat{\boldsymbol{\mu}}_{A} \equiv \hat{\boldsymbol{\mu}}^{0 \alpha}$ and $\hat{\mu}_{B}$ $\equiv \hat{\mu}^{\beta 0}$. Further, $f$ signifies the final electronic state of molecule $\xi, i$ the initial state and $r, s$ are intermediates. [Note, in comparison to Refs. 46 and 47, the indices of $S_{l k}^{\xi}(k)$ $\equiv \alpha_{l k}^{\xi}(k)$ in Eq. (2.1) are interchanged and the structure of $T_{i j l}^{\xi}(k) \equiv \beta_{i j l}^{\xi}(k)$ in Eq. (2.3) differs slightly. The expressions given here are now our preferred form.] To a good approximation, let it be assumed that the state summations in Eqs. (2.2) and (2.3) are limited to the three states that determine the most prominent optical features. These are denoted $|0\rangle$, $|\sigma\rangle,|\alpha\rangle$ for donor $A$, as depicted in Figs. 4 and 5, and $|0\rangle,|\tau\rangle$, $|\beta\rangle$ for acceptor $B$-where $|\alpha\rangle$ and $|\beta\rangle$ are the levels between which energy transfer occurs. It is also expedient to select an input radiation frequency that has a resonance offset with respect to the positioning of these levels, a condition expressible as $E_{\sigma \alpha}=\hbar c k+\Delta E_{A}$, where $\Delta E_{A}$ is a nonzero energy with significantly lower magnitude than a typical transition energy. An expression of similar form, $E_{\tau \beta}=\hbar c k+\Delta E_{B}$, is assumed for $B$. Applying these conditions results in just one summand (or a small number) being significantly larger in magnitude than the rest.

Returning to Eq. (2.1), the part that involves terms comprising $S_{i j}^{\xi}( \pm k)$ quickly simplifies to

$$
\begin{aligned}
\left.M_{f i}^{(4)}\right|_{1}= & \left(\frac{n \hbar c k\left(\mathbf{e} \cdot \boldsymbol{\mu}^{\sigma \alpha}\right)\left(\overline{\mathbf{e}} \cdot \boldsymbol{\mu}^{\beta \tau}\right)}{8 \pi \varepsilon_{0}^{2} V R^{3} \Delta E_{A} \Delta E_{B}}\right) \\
& \times\left(\boldsymbol{\mu}^{0 \sigma} \cdot \boldsymbol{\mu}^{\tau 0}-3\left(\boldsymbol{\mu}^{0 \sigma} \cdot \hat{\mathbf{R}}\right)\left(\boldsymbol{\mu}^{\tau 0} \cdot \hat{\mathbf{R}}\right)\right) .
\end{aligned}
$$

The second contribution to Eq. (2.1), which entails compo- 


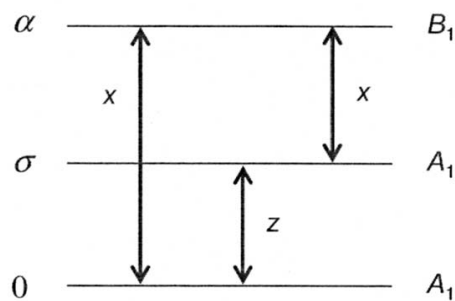

(a)

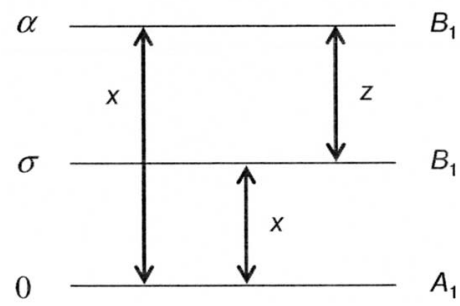

(b)

FIG. 5. As Fig. 4 caption, but for point group symmetry $C_{2 v}$.

nents of the third rank tensor $T_{i j l}^{\xi}(k)$, is found in a similar manner though the conventional analysis is considerably more complex. As proven in detail elsewhere, ${ }^{57}$ a reformulation algorithm expedites the development. The first stage introduces the substitutions $\boldsymbol{\mu}^{\sigma \sigma} \rightarrow \boldsymbol{\mu}^{\sigma \sigma}-\boldsymbol{\mu}^{00} \equiv \breve{\mathbf{d}}, \quad \boldsymbol{\mu}^{\tau \tau} \rightarrow \boldsymbol{\mu}^{\tau \tau}$ $-\boldsymbol{\mu}^{00} \equiv \breve{\mathbf{d}}^{\prime}$ and $\boldsymbol{\mu}^{00}=0$, leaving transition dipole moments unchanged. Limiting the intermediate states of $A$ and $B$ to one of three states as specified above, the possible state sequences are (for $A$ ); $000 \alpha, 00 \sigma \alpha, 0 \sigma 0 \alpha, 00 \alpha \alpha, 0 \alpha 0 \alpha$, $0 \sigma \alpha \alpha, 0 \alpha \sigma \alpha, 0 \sigma \sigma \alpha, 0 \alpha \alpha \alpha$, where for example $0 \sigma 0 \alpha$ represents $\boldsymbol{\mu}^{0 \sigma} \boldsymbol{\mu}^{\sigma 0} \boldsymbol{\mu}^{0 \alpha}$. Every sequence that includes the segment 00 is then discarded; which leaves $0 \sigma 0 \alpha, 0 \alpha 0 \alpha, 0 \sigma \alpha \alpha$, $0 \alpha \sigma \alpha, 0 \sigma \sigma \alpha, 0 \alpha \alpha \alpha$. An analogous set of sequences applies to $B$. Taking the most significant terms that result from Eq. (2.3), the second part of Eq. (2.1) becomes

$$
\begin{aligned}
\left.M_{f i}^{(4)}\right|_{2}= & \left(\frac{n \hbar c k e_{i} \bar{e}_{l}\left(\delta_{j k}-3 \hat{R}_{j} \hat{R}_{k}\right)}{8 \pi \varepsilon_{0}^{2} V R^{3}}\right)\left[\frac { \mu _ { k } ^ { \beta 0 } } { \Delta E _ { A } } \left\{\frac{\mu_{l}^{0 \sigma} \breve{d}_{j} \mu_{i}^{\sigma \alpha}}{\left(E_{\sigma 0}-\hbar c k\right)}\right.\right. \\
& \left.+\frac{\mu_{l}^{0 \alpha} \mu_{j}^{\alpha \sigma} \mu_{i}^{\sigma \alpha}}{\left(E_{\alpha 0}-\hbar c k\right)}+\frac{\mu_{j}^{0 \sigma} \breve{d}_{l} \mu_{i}^{\sigma \alpha}}{E_{\sigma \alpha}}\right\}+\frac{\mu_{k}^{0 \alpha}}{\Delta E_{B}} \\
& \left.\times\left\{\frac{\mu_{l}^{\beta \tau} \breve{d}_{j}^{\prime} \mu_{i}^{\tau 0}}{\left(E_{\tau 0}-\hbar c k\right)}+\frac{\mu_{l}^{\beta \tau} \mu_{j}^{\tau \beta} \mu_{i}^{\beta 0}}{\left(E_{\beta 0}-\hbar c k\right)}+\frac{\mu_{l}^{\beta \tau} \breve{d}_{i}^{\prime} \mu_{j}^{\tau 0}}{E_{\tau \beta}}\right\}\right] .
\end{aligned}
$$

It is worth observing that terms in which intermediate states of the system (molecules plus radiation field) exactly identify with either the initial or final states are specifically excluded in the state summations. Subject to the three-level assumption, the result of Eq. (2.5) delivered by the reformulation procedure is exact.

Next, to proceed to the geometric conditions, we introduce a Cartesian basis in which the donor-acceptor displacement vector $\mathbf{R}$ is identified with the $z$ direction. This signifies that fixed-orientation donor and acceptor molecules should belong to symmetry point groups in which the $x$ and $y$ translations transform under different irreducible representations,

\begin{tabular}{|c|c|c|c|c|}
\hline \multirow{3}{*}{$\begin{array}{c}\text { Transition } \\
\text { dipole moment }\end{array}$} & \multicolumn{4}{|c|}{ Point group } \\
\hline & \multicolumn{2}{|c|}{$D_{2}$} & \multicolumn{2}{|c|}{$C_{2 v}$} \\
\hline & $\Gamma(\sigma)=B_{1}$ & $\Gamma(\sigma)=B_{2}$ & $\Gamma(\sigma)=A_{1}$ & $\Gamma(\sigma)=B_{1}$ \\
\hline \multicolumn{5}{|c|}{ Donor molecule } \\
\hline$\hat{\boldsymbol{\mu}}^{0 \sigma}$ & $\hat{\mathbf{k}}$ & $\hat{\mathbf{j}}$ & $\hat{\mathbf{k}}$ & $\hat{\mathbf{i}}$ \\
\hline$\hat{\boldsymbol{\mu}}^{\sigma \alpha}$ & $\hat{\mathbf{j}}$ & $\hat{\mathbf{k}}$ & $\hat{\mathbf{i}}$ & $\hat{\mathbf{k}}$ \\
\hline \multicolumn{5}{|c|}{ Acceptor molecule } \\
\hline$\hat{\boldsymbol{\mu}}^{r 0}$ & $\hat{\mathbf{k}}$ & $-\hat{\mathbf{i}}$ & $\hat{\mathbf{k}}$ & $\hat{\mathbf{j}}$ \\
\hline$\hat{\boldsymbol{\mu}}^{\beta \tau}$ & $-\hat{\mathbf{i}}$ & $\hat{\mathbf{k}}$ & $\hat{\mathbf{j}}$ & $\hat{\mathbf{k}}$ \\
\hline
\end{tabular}

TABLE I. List of the relevant transition dipole moments and their orientations for the donor and acceptor.

such that $\hat{\boldsymbol{\mu}}_{A} \equiv \hat{\boldsymbol{\mu}}^{0 \alpha}$ and $\hat{\boldsymbol{\mu}}_{B} \equiv \hat{\boldsymbol{\mu}}^{\beta 0}$ can be chosen unambiguously as being directed in the $\hat{\mathbf{i}}$ and $\hat{\mathbf{j}}$ directions, respectively. It transpires that the only symmetry classes to satisfy such a specification are the well-known Schoenflies groups $D_{2}$, for which $y$-directed vectors transform under the $B_{2}$ representation and $x$ under $B_{3}$, and $C_{2 v}$-the latter with $B_{1}(x)$ and $B_{2}(y)$.

First taking $D_{2}$, the representation $\Gamma(\alpha)$ of the donor final state will usually be $B_{3}$. The reason is that the ground state representation is generally totally symmetric (i.e., $\Gamma(0)=A$ ) and $\hat{\boldsymbol{\mu}}^{0 \alpha}=\hat{\mathbf{i}}$ corresponds to $B_{3}$ in the character tables; the direct product of these two state representations provides the symmetry of the resultant excited state. Another requirement is that the same representation is contained in the direct product of the representations of $\hat{\boldsymbol{\mu}}^{0 \sigma}$ and $\hat{\boldsymbol{\mu}}^{\sigma \alpha}$; hence $\Gamma(\sigma)$ must be of $B_{1}$ or $B_{2}$ symmetry (see Fig. 4). For example when $\Gamma(\sigma)=B_{1}$, the transition vector representations are $A \otimes B_{1}=B_{1}$ and $B_{1} \otimes B_{3}=B_{2}$ and the direct product $B_{1}$ $\otimes B_{2}=B_{3}$, as required. These calculations imply $\hat{\boldsymbol{\mu}}^{0 \sigma}=\hat{\mathbf{k}}$ and $\hat{\boldsymbol{\mu}}^{\sigma \alpha}=\hat{\mathbf{j}}$. For $\Gamma(\sigma)=B_{2}$ similar considerations determine that $\hat{\boldsymbol{\mu}}^{0 \sigma}=\hat{\mathbf{j}}$ and $\hat{\boldsymbol{\mu}}^{\sigma \alpha}=\hat{\mathbf{k}}$.

Applying the same procedure for $C_{2 v}$, results are obtained as illustrated in Fig. 5 and contained within Table I. Moreover, the orientations of $\breve{\mathbf{d}}$ and $\breve{\mathbf{d}}^{\prime}$ will be such that they belong to the totally symmetric representation, as the latter is always included in the direct product of identical (nondegenerate) state representations. Therefore, both $\breve{\mathbf{d}}$ and $\breve{\mathbf{d}}^{\prime}$ point in the $z$ direction for $C_{2 v}$ (note, there is no such result for $D_{2}$ ). Due to the mutual orthogonality conditions for OCRET, the dipole orientations of the acceptor are easily determined by taking these vector results and rotating each by $90^{\circ}$ around the $z$ axis (see Fig. 6). For convenience $A$ and $B$ are chosen to be symmetrically identical, although this calculational method allows for systems where this is not necessarily the case. A complete list of the relevant transition dipole moments and their orientations is given in Table I.

Returning to Eq. (2.4) and using the above symmetry information, it now becomes clear that the only nonzero result for the $D_{2}$ case will be with $\Gamma(\sigma)=B_{1}$, and in the $C_{2 v}$ case, $\Gamma(\sigma)=A_{1}$. Therefore, for these symmetries, Eq. (2.4) is rewritten as 


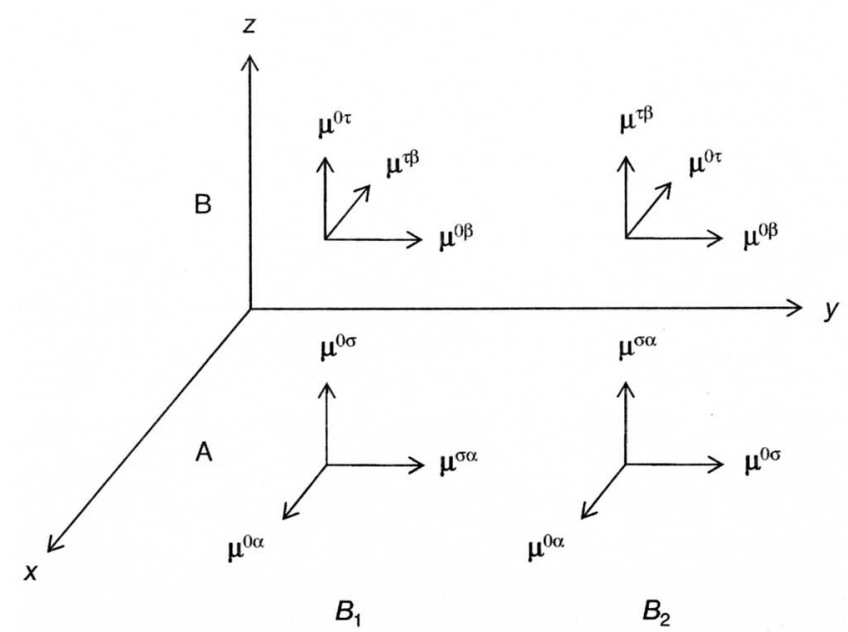

(a)

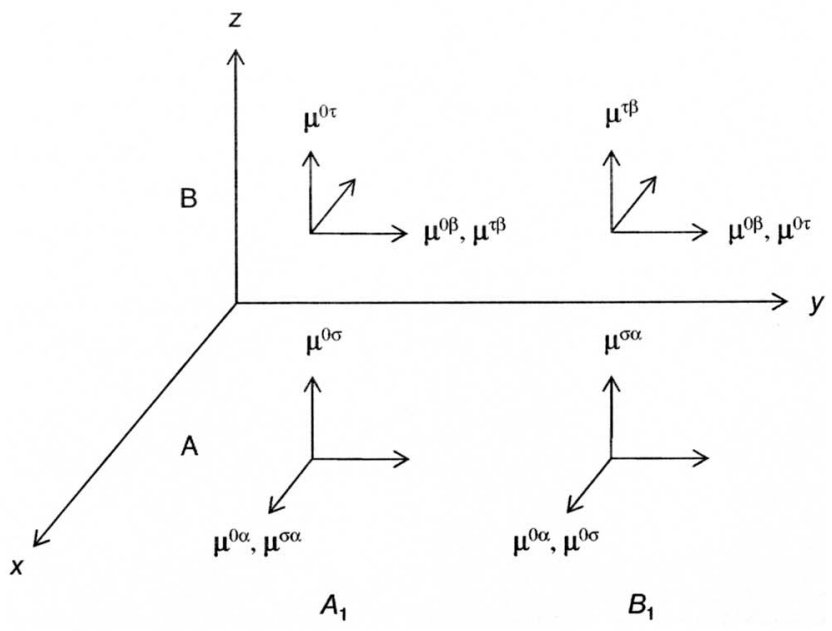

(b)

FIG. 6. Orientations of the relevant transition dipole moments for both donor $A$ and acceptor $B$, determined for each of the possible intermediate state symmetries. These are illustrated for the point groups: (a) $D_{2}$ and (b) $C_{2 v}$.

$$
\left.M_{f i}^{(4)}\right|_{1}=\left(\frac{n \hbar c k|\boldsymbol{\mu}|^{4}}{8 \pi \varepsilon_{0}^{2} V r^{3} \Delta E_{A} \Delta E_{B}}\right) \Phi_{n}^{m} \kappa^{\prime},
$$

where the orientation factor is $\kappa^{\prime}=\left(R^{2}-3 r^{2}\right) / R^{2}=-2$, given that $\mathbf{R}=r \hat{\mathbf{k}}$, in which $r$ is the displacement of $B$ from $A$ and is of sufficient magnitude to allow for the dielectric isolation of the two molecules. Further, $\Phi_{C_{2 v}}^{A_{1}}=\sin ^{2} \phi \cos \theta \sin \theta$ and $\Phi_{D_{2}}^{B_{1}}=-\Phi_{C_{2 v}}^{A_{1}}$, which are determined from the orientation of $\mathbf{e}$ in spherical coordinates (with the input photon polarization assumed linear); moreover the subscript $n$ and superscript $m$ denote the symmetry point group and intermediate state symmetry, respectively. In addition, all transition dipole moments will have similar magnitude and, thus, may be assumed equivalent; similarly $\Delta E_{A}$ and $\Delta E_{B}$ may be assumed to have similar values, so that their subscripts can be dropped. Analyzing the contribution of Eq. (2.5) to $M_{f i}^{(4)}$, it becomes apparent that only $D_{2}$ with $\Gamma(\sigma)=B_{1}$ will be of significance. This is a consequence of the fact that $\boldsymbol{\mu}^{\beta 0} \| \boldsymbol{\mu}^{\alpha \sigma}$ and $\boldsymbol{\mu}^{\tau \beta} \| \boldsymbol{\mu}^{0 \alpha}$. With this in mind Eq. (2.5) gives

$$
\left.M_{f i}^{(4)}\right|_{2}=\left(\frac{n \hbar c k|\boldsymbol{\mu}|^{4}}{4 \pi \varepsilon_{0}^{2} V r^{3}}\right)\left(\frac{-\Phi_{D_{2}}^{B_{1}}}{\Delta E\left(E_{\alpha 0}-\hbar c k\right)}\right),
$$

since $E_{\beta 0}=E_{\alpha 0}$ due to energy conservation. Therefore a complete quantum amplitude expression is found by the addition of Eqs. (2.6) and (2.7) to give

$$
M_{f i}^{(4)}=\left(\frac{-n \hbar c k|\boldsymbol{\mu}|^{4}}{4 \pi \varepsilon_{0}^{2} V r^{3} \Delta E}\right)\left(\frac{\Phi_{n}^{m}}{\Delta E}+\frac{\Phi_{D_{2}}^{B_{1}}}{E_{\alpha 0}-\hbar c k}\right),
$$

which, unlike the result for spontaneous RET under these conditions, is clearly a nonzero result. The corresponding time-dependent probability $P(t)$ for energy transfer is delivered by the Fermi golden rule, ${ }^{58}$ namely,

$$
P(t)=\frac{2 \pi \rho_{f}}{\hbar} \int_{0}^{t}\left|M_{f i}^{(2)}+M_{f i}^{(4)}+\cdots\right|^{2} \mathrm{~d} t,
$$

with higher-order quantum amplitudes rapidly diminishing in magnitude. In Eq. (2.9), $M_{f i}^{(2)}$ corresponds to RET-a null quantity in this case - and $\rho_{f}$ is the density of states. Inserting Eq. (2.8) into (2.9) gives the expression

$$
\begin{aligned}
P(t)= & \frac{4 K C^{2} J^{\prime}}{r^{6}}\left\{\left(\frac{\Phi_{n}^{m}}{\Delta E}\right)^{2}+\left(\Phi_{D_{2}}^{B_{1}}\right)^{2}\left(\frac{2}{\Delta E\left(E_{\alpha 0}-\hbar c k\right)}\right.\right. \\
& \left.\left.+\left(\frac{1}{E_{\alpha 0}-\hbar c k}\right)^{2}\right)\right\},
\end{aligned}
$$

where the variables are defined as $K=|\boldsymbol{\mu}|^{4} \rho_{f} / 8 \pi \varepsilon_{0}^{2} \hbar, C$ $=|\boldsymbol{\mu}|^{2} / 2 c \varepsilon_{0} \Delta E$, and $J^{\prime}=\int_{0}^{t} I^{2}(t) \mathrm{d} t$, in which $I(t) \equiv n \hbar c^{2} k / V$ is the throughput laser irradiance. It is notable that with $\phi$ $=0^{\circ}, \theta=0^{\circ}$, or $\theta=90^{\circ}$, no energy transfer to the acceptor occurs.

\section{ONE-DIMENSIONAL LINEAR ARRAYS}

To develop the model of the last section into a potentially workable all-optical switch, we first investigate the case of two one-dimensional linear arrays-one a donor array and the other an acceptor array-each comprising a set of equally spaced, identical and optically distinct molecules. The donor species are chemically different from the acceptor species, to preclude back transfer. ${ }^{59}$ The two arrays are constructed so that each constituent molecule of the donor array directly corresponds to a molecule within the acceptor array; these pairs are given the coordinate $u$ (an integer value) and the acceptor is displaced from its counterpart donor by $r \hat{\mathbf{k}}$. Further, all molecules in the donor and acceptor arrays are orientated in the $\hat{\mathbf{i}}$ and $\hat{\mathbf{j}}$ directions, respectively, in order that the initial condition of OCRET (preclusion of spontaneous RET) is satisfied. The geometry is illustrated by Fig. 7.

We now address the capacities for energy transfer from an excited donor (for convenience at $u=0$ ) to any other molecules within either array. First, consider energy relocation to an arbitrary molecule within the acceptor array. The relevant quantum amplitude is found by the same process as discussed in the preceding section. Again, RET is precluded by the geometric configuration. As $\mathbf{R}=u \hat{\mathbf{i}}+r \hat{\mathbf{k}}$ in this instance, where $l$ is the lattice constant, Eq. (2.4) becomes 


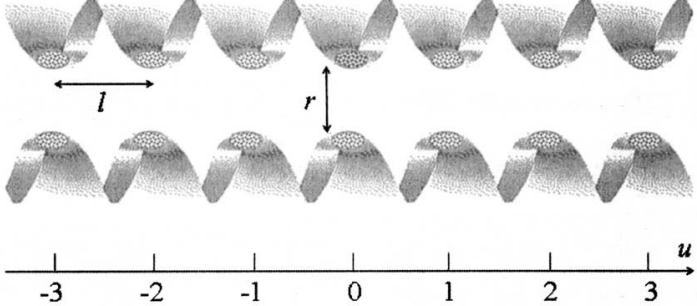

FIG. 7. Graphical depiction of parallel one-dimensional linear-lattice arrays Here, $l$ represents the lattice constant and $r$ the displacement of the upper donor array from the lower acceptor array. The optically active molecules, each labeled with an integer coordinate, are denoted by pale ellipses (ground state) or a dark ellipse (excited state).

$$
\left.M_{f i}^{(4)}\right|_{1}=\left(\frac{n \hbar c k|\boldsymbol{\mu}|^{4}}{8 \pi \varepsilon_{0}^{2} l^{3} V(\Delta E)^{2}}\right)\left(\frac{u^{2}-2 r^{\prime 2}}{\left(u^{2}+r^{\prime 2}\right)^{5 / 2}}\right) \Phi_{n}^{m},
$$

since $\kappa^{\prime}=\left(u^{2}-2 r^{\prime 2}\right) /\left(u^{2}+r^{\prime 2}\right)$, in which $r^{\prime}=r / l$ is the aspect ratio. This result, Eq. (3.1), applies to either molecules of $D_{2}$ symmetry, where $\Gamma(\sigma)=B_{1}$, or $C_{2 v}$ with $\Gamma(\sigma)=A_{1}$. In contrast the exact expressions for the other contributions to the quantum amplitude, those corresponding to Eq. (2.5), depend significantly on the symmetries. For $D_{2}$ molecules with $\Gamma(\sigma)=B_{1}$, all terms containing either $\breve{\mathbf{d}}$ and $\breve{\mathbf{d}^{\prime}}$ are excluded, as a consequent of the argument detailed in Sec. II, to give the expression

$$
\left.M_{f i}^{(4)}\right|_{2}=\left(\frac{n \hbar c k|\boldsymbol{\mu}|^{4} \Phi_{D_{2}}^{B_{1}}}{8 \pi \varepsilon_{0}^{2} l^{3} V \Delta E}\right)\left(\frac{u^{2}-2 r^{\prime 2}}{\left(u^{2}+r^{\prime 2}\right)^{5 / 2}}\right)\left(\frac{1}{E_{\alpha 0}-\hbar c k}\right) .
$$

For the point group of $C_{2 v}$ with $\Gamma(\sigma)=A_{1}$ the following emerges:

$$
\begin{aligned}
\left.M_{f i}^{(4)}\right|_{2}= & \left(\frac{-n \hbar c k|\boldsymbol{\mu}|^{4} \breve{\Phi}_{C_{2 v}}^{A_{1}}}{8 \pi \varepsilon_{0}^{2} l^{3} V \Delta E}\right)\left(\frac{3 u r^{\prime}}{\left(u^{2}+r^{\prime 2}\right)^{5 / 2}}\right)\left(\frac{1}{\Delta E+E_{\alpha 0}}\right. \\
& \left.+\frac{1}{\Delta E+\hbar c k}\right),
\end{aligned}
$$

where $\breve{\Phi}_{C_{2 v}}^{A_{1}}=\sin \phi \cos \phi \sin \theta$. A complete expression is thus found from Eq. (3.1), and either Eqs. (3.2) or (3.3), to give

$$
M_{f i}^{(4)}=\left(\frac{n \hbar c k|\boldsymbol{\mu}|^{4}}{8 \pi \varepsilon_{0}^{2} l^{3} V \Delta E}\right)\left(\frac{1}{\left(u^{2}+r^{\prime 2}\right)^{5 / 2}}\right) F_{n}^{m}
$$

where $F_{n}^{m}$ refers to either of the two applied symmetries and is explicitly written as

$$
\begin{aligned}
F_{D_{2}}^{B_{1}}= & \Phi_{D_{2}}^{B_{1}}\left(u^{2}-2 r^{\prime 2}\right)\left(\frac{1}{\Delta E}+\frac{1}{E_{\alpha 0}-\hbar c k}\right), \\
F_{C_{2 v}}^{A_{1}}= & \frac{\Phi_{C_{2 v}}^{A_{1}}\left(u^{2}-2 r^{\prime 2}\right)}{\Delta E}-3 u r^{\prime} \breve{\Phi}_{C_{2 v}}^{A_{1}}\left(\frac{1}{\Delta E+E_{\alpha 0}}\right. \\
& \left.+\frac{1}{\Delta E+\hbar c k}\right) .
\end{aligned}
$$

It is readily verified that Eq. (3.4) reduces to (2.8) (the result for a directly opposing donor-acceptor pair) on setting $u=0$.

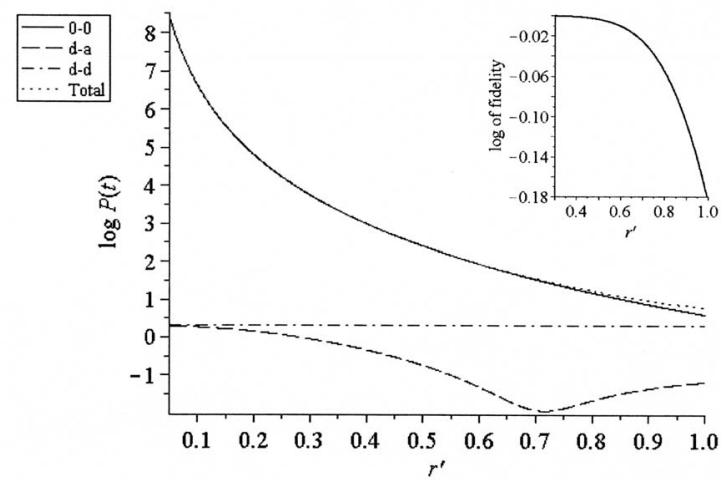

FIG. 8. Plot of $\log P(t)$, where $P(t)$ is the time-dependent probability, against the aspect ratio $r^{\prime}$ for optical transfer from an excited molecule in the donor linear array to the required destination in the acceptor linear array (0-0); also depicted are the "cross-talk" probabilities for transfer to another molecule in either the acceptor $(d-a)$ or the donor $(d-d)$ array, and the sum of all three transfer possibilities (total). (Inset) difference between logarithms of the $0-0$ and the sum probabilities for various $r^{\prime}$, signifying on a logarithmic scale the transfer fidelity; on the ordinate axis each -0.01 increment corresponds to $2.3 \%$ loss.

Inserting Eq. (3.4) into (2.9) gives the following general expression for OCRET between a given donor and an arbitrary acceptor molecule:

$$
P(t)=\frac{K C^{2} J^{\prime}}{l^{6}\left(u^{2}+r^{\prime 2}\right)^{5}}\left(F_{n}^{m}\right)^{2} .
$$

The second case to consider is a transfer of energy from one initially excited donor molecule to another within the donor array. The resulting expression is analogous to Eq. (3.7) but with $r^{\prime}$ taken as zero. Indeed, with $\mathbf{R}=u \hat{\mathbf{j}}+r \hat{\mathbf{k}}$ the only difference from above is that $\breve{\Phi}_{C_{2 v}}^{A_{1}}$ is replaced by $\hat{\Phi}_{C_{2 v}}^{A_{1}}$ $=\sin \phi \cos \phi \cos \theta$.

To quantify the twin-array transfer fidelity we now compare the efficiency of direct energy relocation, from an excited donor to its designated partner in the acceptor array, to the summed efficiencies for transfer to any other molecules within either array. From calculations based on typical parameter values $\Delta E=3 \times 10^{-20} \mathrm{~J},|\boldsymbol{\mu}|=1 \times 10^{-29} \mathrm{C} \mathrm{m}$, and with $\phi=90^{\circ}$ and $\theta=45^{\circ}$-noting that these variables affect the absolute transfer efficiency but not the destination of the excitation-the dependence of the result on the aspect ratio is determined and graphically depicted as plots of $P(t)$ for different transfer destinations, shown in Fig. 8. In this representation, energy transfer outside the range defined by the limits $u= \pm 10$ is negligible. Moreover only the first terms of Eqs. (3.5) and (3.6) contribute significantly [as they dominate through their $(\Delta E)^{-2}$ dependence] resulting in identical outcomes of Eq. (3.7) for both of the admissible molecular symmetries. As the plots within Fig. 8 illustrate, the transfer of energy from the excited donor to the corresponding molecule in the acceptor array greatly dominates all other transfer routes for any aspect ratio up to about 0.5 . Specifically, for array geometries that fall into this category, no less than $98.9 \%$ of the total excitation is transported to the required destination. As can be anticipated, this excitation fidelity is increasingly compromised if the aspect ratio is increased; when $r^{\prime}=1$, cross-talk to other molecules becomes a major 


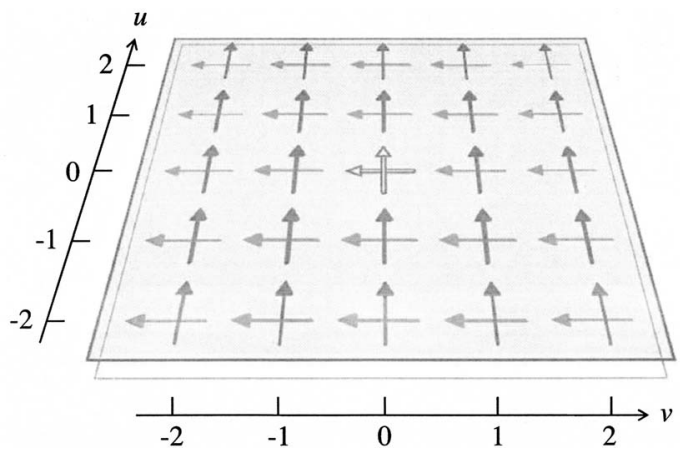

FIG. 9. Structure of the two-dimensional square-lattice arrays, viewed from above. Both lie in the $i j$ plane, with all donor transition moments (black) in the upper array parallel to the $i$ axis, and all acceptor transition moments (gray) in the lower array parallel to the $j$ axis. The open arrows represent one excited donor and its counterpart acceptor. By reducing both arrays to a single row or column an equivalent graphical representation to Fig. 7 is found.

factor, with a $35.4 \%$ loss of the intended transfer-see Fig. 8 (inset). Notably, unlike the case of the following twodimensional systems, the destination of the initial excitation is not dependent on the laser intensity. Results from this section clearly show that, with favorable $r^{\prime}$ values, linear arrays represent tenable components of a parallel-operation all-optical switch. To consider ways of achieving the best use of space, however, we now turn attention to two-dimensional arrays, the subject of the next two sections.

\section{TWO-DIMENSIONAL SQUARE-LATTICE ARRAYS}

A system that appears to offer a higher information processing density is an all-optical switch based on a pair of two-dimensional arrays, and we first consider a square-lattice arrangement (Fig. 9). To develop theory for this system the linear arrays of the previous section are extended into two dimensions - so that each molecule within an array is now labeled $(u, v)$, where $u$ and $v$ are integers. The transfer displacement vector, signifying the position of an arbitrary acceptor relative to a donor at the (nominal) origin within the donor plane, is $\mathbf{R}=u \hat{\mathbf{i}}+v l \hat{\mathbf{j}}+r \hat{\mathbf{k}}$. In this case spontaneous RET is no longer entirely absent, since it may occur between the donor and any off-axis molecules, ${ }^{42}$ i.e., where $u \neq 0$ and $v \neq 0$. To find an expression for $P(t)$ for this system the following equation, determined in earlier RET work, ${ }^{60}$ is required [note, within Ref. 60 the modulus squared should be omitted from the left-hand side of Eq. 5.10]:

$$
M_{f i}^{(2)} \bar{M}_{f i}^{(2)}=\left(\frac{\left|\boldsymbol{\mu}_{A}\right|^{2}\left|\boldsymbol{\mu}_{B}\right|^{2} \kappa^{2}}{16 \pi^{2} \varepsilon_{0}^{2} R^{6}}\right)
$$

where $\kappa=\cos \theta-3 \cos \phi \cos \gamma$ (with $\theta$ the angle between $\left|\boldsymbol{\mu}_{A}\right|$ and $\left|\boldsymbol{\mu}_{B}\right|$, and $\phi, \gamma$ the angles between $\mathbf{R}$ and $\left|\boldsymbol{\mu}_{A}\right|,\left|\boldsymbol{\mu}_{B}\right|$ respectively). Hence, for on-axis molecules $\theta=90^{\circ}$, and $\phi$ $=90^{\circ}$ or $\gamma=90^{\circ}$; therefore $\kappa=0$ and spontaneous RET is precluded. In application to this system Eq. (4.1) becomes

$$
\left|M_{f i}^{(2)}\right|^{2}=\left(\frac{|\boldsymbol{\mu}|^{4}}{16 \pi^{2} \varepsilon_{0}^{2} l^{6}}\right)\left(\frac{9 u^{2} v^{2}}{\left(u^{2}+v^{2}+r^{\prime 2}\right)^{5}}\right)
$$

since $\kappa=-3 u v /\left(u^{2}+v^{2}+r^{\prime 2}\right)$. The transfer contribution due to the OCRET quantum amplitude is again determined in two parts, the first being developed from Eq. (3.1),

$$
\left.M_{f i}^{(4)}\right|_{1}=\left(\frac{n \hbar c k|\boldsymbol{\mu}|^{4}}{8 \pi \varepsilon_{0}^{2} l^{3} V(\Delta E)^{2}}\right)\left(\frac{u^{2}+v^{2}-2 r^{\prime 2}}{\left(u^{2}+v^{2}+r^{\prime 2}\right)^{5 / 2}}\right) \Phi_{n}^{m},
$$

as $\kappa^{\prime}=\left(u^{2}+v^{2}-2 r^{\prime 2}\right) /\left(u^{2}+v^{2}+r^{\prime 2}\right)$ for both $D_{2}$ with $\Gamma(\sigma)$ $=B_{1}$, and $C_{2 v}$ with $\Gamma(\sigma)=A_{1}$. For $D_{2}$ symmetry, the second part of the quantum amplitude is an obvious generalization of its analogous linear-array expression (3.2), and is thus,

$$
\begin{aligned}
\left.M_{f i}^{(4)}\right|_{2}= & \left(\frac{n \hbar c k|\boldsymbol{\mu}|^{4} \Phi_{D_{2}}^{B_{1}}}{8 \pi \varepsilon_{0}^{2} l^{3} V \Delta E}\right)\left(\frac{u^{2}+v^{2}-2 r^{\prime 2}}{\left(u^{2}+v^{2}+r^{\prime 2}\right)^{5 / 2}}\right) \\
& \times\left(\frac{1}{E_{\alpha 0}-\hbar c k}\right) .
\end{aligned}
$$

In contrast, the second part for the $C_{2 v}$ point group is much more intricate than the corresponding Eq. (3.3), and the result is as follows:

$$
\begin{aligned}
\left.M_{f i}^{(4)}\right|_{2}= & \left(\frac{-3 n \hbar c k|\boldsymbol{\mu}|^{4}}{8 \pi \varepsilon_{0}^{2} l^{3} V \Delta E\left(u^{2}+v^{2}+r^{\prime 2}\right)^{5 / 2}}\right) \\
& \times\left(r^{\prime}\left(\hat{\Phi}_{C_{2 v}}^{A_{1}} v+\breve{\Phi}_{C_{2 v}}^{A_{1}} u\right)\left(\frac{1}{\Delta E+E_{\alpha 0}}+\frac{1}{\Delta E+\hbar c k}\right)\right. \\
& \left.+\frac{u v \sin ^{2} \phi}{E_{\alpha 0}-\hbar c k}\right) .
\end{aligned}
$$

The full expression for the quantum amplitude due to OCRET is a sum of the expressions given by Eq. (4.3), and either Eqs. (4.4) or (4.5) as appropriate, giving

$$
M_{f i}^{(4)}=\left(\frac{n \hbar c k|\boldsymbol{\mu}|^{4}}{8 \pi \varepsilon_{0}^{2} l^{3} V \Delta E}\right)\left(\frac{1}{\left(u^{2}+v^{2}+r^{\prime 2}\right)^{5 / 2}}\right) F_{n}^{m},
$$

with $F_{n}^{m}$ written explicitly as

$$
\begin{aligned}
F_{D_{2}}^{B_{1}}= & \Phi_{D_{2}}^{B_{1}}\left(u^{2}+v^{2}-2 r^{\prime 2}\right)\left(\frac{1}{\Delta E}+\frac{1}{E_{\alpha 0}-\hbar c k}\right) \\
F_{C_{2 v}}^{A_{1}}= & \frac{\Phi_{C_{2 v}}^{A_{1}}\left(u^{2}+v^{2}-2 r^{\prime 2}\right)}{\Delta E} \\
& -3\left(r^{\prime}\left(\hat{\Phi}_{C_{2 v}}^{A_{1}} v+\breve{\Phi}_{C_{2 v}}^{A_{1}} u\right)\left(\frac{1}{\Delta E+E_{\alpha 0}}+\frac{1}{\Delta E+\hbar c k}\right)\right. \\
& \left.+\frac{u v \sin ^{2} \phi}{E_{\alpha 0}-\hbar c k}\right) .
\end{aligned}
$$

Employing Eqs. (4.2) and (4.6) with (2.9), the following result is then secured for the transfer probability at time $t$ : 


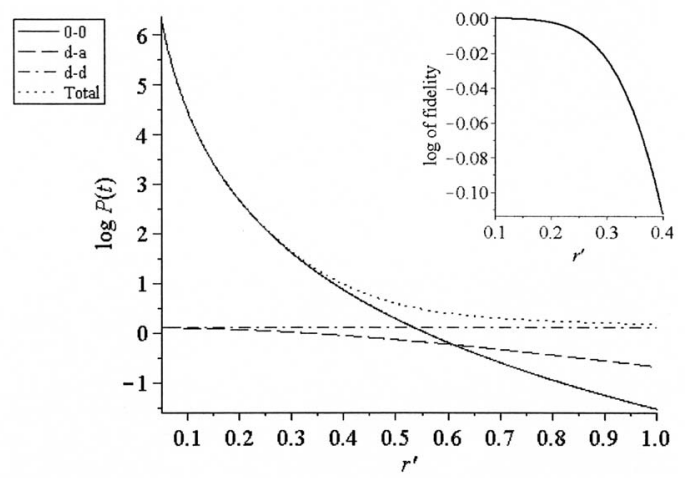

FIG. 10. Graph illustrating $\log P(t)$ against $r^{\prime}$ for pair of two dimensional square arrays. Here, the irradiance $I$ of the input laser is $1 \times 10^{12} \mathrm{~W} \mathrm{~cm}^{-2}$ and the key is that of Fig. 8 .

$$
\begin{aligned}
P(t)= & \frac{K}{l^{6}\left(u^{2}+v^{2}+r^{\prime 2}\right)^{5}}\left[9 u^{2} v^{2} t-6 u v C J F_{n}^{m}\right. \\
& \left.+C^{2} J^{\prime}\left(F_{n}^{m}\right)^{2}\right]
\end{aligned}
$$

correct to fourth-order perturbation theory, and where $J$ $=\int_{0}^{\mathrm{t}} I(t) \mathrm{d} t$ is the energy fluence of the laser input.

Once again, graphical depictions of $P(t)$ for various transfer destinations are used to exhibit and compare the probabilities for the intended energy transfer to the excited donor's counterpart acceptor, in contrast to the cross-talk possibilities represented by unsought routing of the excitation to other molecules. Both the aspect ratio and the laser irradiance prove to exert a bearing on the form of resultssee Figs. 10 and 11. The mapping fidelity, denoting the fraction of the transfer that delivers energy to the counterpart acceptor, is plotted in Figs. 10 and 11 (insets). In performing the calculations for these graphs, just the first terms of Eqs. (4.7) and (4.8) are taken (consistent with the linear array case); energy transfers for which $|u|,|v|>10$ prove negligible. The calculations are performed with the data $\Delta E=3$ $\times 10^{-20} \mathrm{~J},|\boldsymbol{\mu}|=1 \times 10^{-29} \mathrm{C} \mathrm{m}, \phi=90^{\circ}$ and $\theta=45^{\circ}$. The laser irradiance, assumed to be time independent, has a value of $1 \times 10^{12}$ and $1 \times 10^{10} \mathrm{~W} \mathrm{~cm}^{-2}$ for Figs. 10 and 11 , respectively.

It is clear that the irradiance is a major contributing factor in the destination of the donor excitation. To achieve transfer losses less than $5 \%$ the aspect ratio $r^{\prime}$ can be little

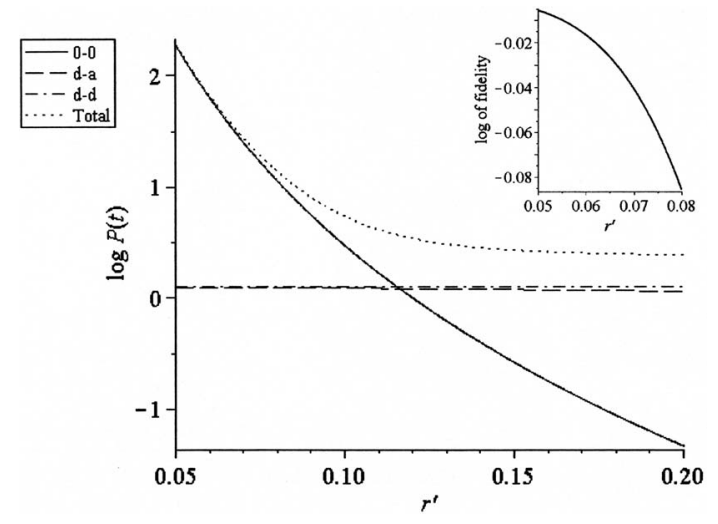

FIG. 11. Graph as Fig. 10, but for $I=1 \times 10^{10} \mathrm{~W} \mathrm{~cm}^{-2}$.

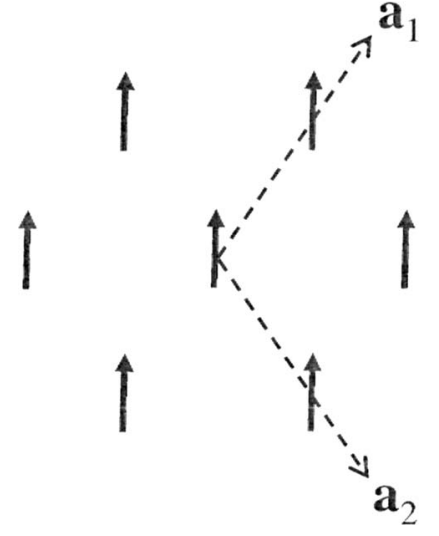

FIG. 12. In-plane coordinate system for a two-dimensional hexagonal lattice.

over 0.3 for $I=1 \times 10^{12} \mathrm{~W} \mathrm{~cm}^{-2}$, or 0.06 for 1 $\times 10^{10} \mathrm{~W} \mathrm{~cm}^{-2}$. These $r^{\prime}$ values are both practicable—but if the latter is to be achieved through an expansion of the lattice constant, it may compromise the potentially miniature dimensions of a device. The next section will determine whether these figures can be improved upon, by examining two-dimensional hexagonal-lattice arrays as potential components in an all-optical switch.

\section{TWO-DIMENSIONAL HEXAGONAL-LATTICE ARRAYS}

In the investigation of an array of two-dimensional hexagonal lattices, it is convenient to choose a coordinate system as illustrated by Fig. 12. For calculations on this system, the donor-acceptor displacement vector $\mathbf{R}=u l \hat{\mathbf{a}}_{1}+v l \hat{\mathbf{a}}_{2}+r \hat{\mathbf{k}}$, as cast in terms of the nonorthogonal lattice unit vectors, is converted into Cartesian coordinates so that

$$
\mathbf{R}=\frac{\sqrt{3} l}{2}(u-v) \hat{\mathbf{i}}+\frac{l}{2}(u+v) \hat{\mathbf{j}}+r \hat{\mathbf{k}} .
$$

In this system spontaneous RET is possible from the donor to all other molecules (except where $u=v$ or $u=-v$ ) as is determined from the following expression:

$$
\left|M_{f l}^{(2)}\right|^{2}=\left(\frac{108|\boldsymbol{\mu}|^{4}}{\pi^{2} \varepsilon_{0}^{2} l^{6}}\right)\left(\frac{(u-v)^{2}(u+v)^{2}}{\left(3(u-v)^{2}+(u+v)^{2}+4 r^{\prime 2}\right)^{5}}\right),
$$

where $\kappa=-3 \sqrt{3}(u-v)(u+v) /\left(3(u-v)^{2}+(u+v)^{2}+4 r^{\prime 2}\right)$. The transfer contribution due to OCRET is resolved by the customary method. The first part is written as

$$
\begin{aligned}
\left.M_{f l}^{(4)}\right|_{1}= & \left(\frac{n \hbar c k|\boldsymbol{\mu}|^{4}}{\pi \varepsilon_{0}^{2} l^{3} V(\Delta E)^{2}}\right) \\
& \times\left(\frac{3(u-v)^{2}+(u+v)^{2}+r^{\prime 2}}{\left(3(u-v)^{2}+(u+v)^{2}+4 r^{\prime 2}\right)^{5 / 2}}\right) \Phi_{n}^{m},
\end{aligned}
$$

since $\kappa^{\prime}=\left(3(u-v)^{2}+(u+v)^{2}+r^{\prime 2}\right) /\left(3(u-v)^{2}+(u+v)^{2}+4 r^{\prime 2}\right)$ for both symmetries. The point group of $D_{2}$ has the second part, 


$$
\begin{aligned}
\left.M_{f l}^{(4)}\right|_{2}= & \left(\frac{n \hbar c k|\boldsymbol{\mu}|^{4} \Phi_{D_{2}}^{B_{1}}}{\pi \varepsilon_{0}^{2} l^{3} V \Delta E}\right) \\
& \times\left(\frac{3(u-v)^{2}+(u+v)^{2}-8 r^{\prime 2}}{\left(3(u-v)^{2}+(u+v)^{2}+4 r^{\prime 2}\right)^{5 / 2}}\right) \\
& \times\left(\frac{1}{E_{\alpha 0}-\hbar c k}\right) .
\end{aligned}
$$

In comparison, the second part is again more intricate for $C_{2 v}$ and given by

$$
\begin{aligned}
\left.M_{f l}^{(4)}\right|_{2}= & \left(\frac{-3 n \hbar c k|\boldsymbol{\mu}|^{4}}{\pi \varepsilon_{0}^{2} l^{3} V \Delta E}\right)\left(2 r ^ { \prime } \left((u+v) \hat{\Phi}_{C_{2 v}}^{A_{1}}\right.\right. \\
& \left.+\sqrt{3}(u-v) \breve{\Phi}_{C_{2 v}}^{A_{1}}\right)\left(\frac{1}{\Delta E+E_{\alpha 0}}+\frac{1}{\Delta E+\hbar c k}\right) \\
& \left.+\sqrt{3}(u-v)(u+v) \frac{\sin ^{2} \phi}{E_{\alpha 0}-\hbar c k}\right)\left(3(u-v)^{2}\right. \\
& \left.+(u+v)^{2}+4 r^{\prime 2}\right)^{-5 / 2} .
\end{aligned}
$$

A full and general expression is determined from Eqs. (5.3)-(5.5) producing

$$
M_{f l}^{(4)}=\left(\frac{n \hbar c k|\boldsymbol{\mu}|^{4} F_{n}^{m}}{\pi \varepsilon_{0}^{2} l^{3} V \Delta E}\right)\left(3(u-v)^{2}+(u+v)^{2}+4 r^{\prime 2}\right)^{-5 / 2},
$$

with $F_{n}^{m}$ written explicitly as

$$
\begin{aligned}
F_{D_{2}}^{B_{1}}= & \Phi_{D_{2}}^{B_{1}}\left(\frac{3(u-v)^{2}+(u+v)^{2}+r^{\prime 2}}{\Delta E}\right. \\
& \left.+\frac{3(u-v)^{2}+(u+v)^{2}-8 r^{\prime 2}}{E_{\alpha 0}-\hbar c k}\right), \\
F_{C_{2 v}}^{A_{1}}= & \frac{\Phi_{C_{2 v}}^{A_{1}}\left(3(u-v)^{2}+(u+v)^{2}+r^{\prime 2}\right)}{\Delta E} \\
& -3\left(2 r^{\prime}\left((u+v) \hat{\Phi}_{C_{2 v}}^{A_{1}}+\sqrt{3}(u-v) \breve{\Phi}_{C_{2 v}}^{A_{1}}\right)\right. \\
& \times\left(\frac{1}{\Delta E+E_{\alpha 0}}+\frac{1}{\Delta E+\hbar c k}\right) \\
& \left.+\sqrt{3}(u-v)(u+v) \frac{\sin ^{2} \phi}{E_{\alpha 0}-\hbar c k}\right) .
\end{aligned}
$$

Through the use of Eqs. (5.2) and (5.6) with Eq. (2.9), the following is found:

$$
\begin{aligned}
P(t)= & \frac{K}{l^{6}}\left[1728(u-v)^{2}(u+v)^{2} t\right. \\
& \left.-384 \sqrt{3} C J F_{n}^{m}(u-v)(u+v)+64 C^{2} J^{\prime}\left(F_{n}^{m}\right)^{2}\right] \\
& \times\left(3(u-v)^{2}+(u+v)^{2}+4 r^{\prime 2}\right)^{-5} .
\end{aligned}
$$

In the same manner as previously, various plots of $P(t)$ are constructed-Figs. 13 and 14. On comparing these graphs it is again evident that the laser intensity plays a major role, as for the case of square-lattice arrays. To achieve transfer losses less than $5 \%$ in the present case, $r^{\prime}$ cannot be

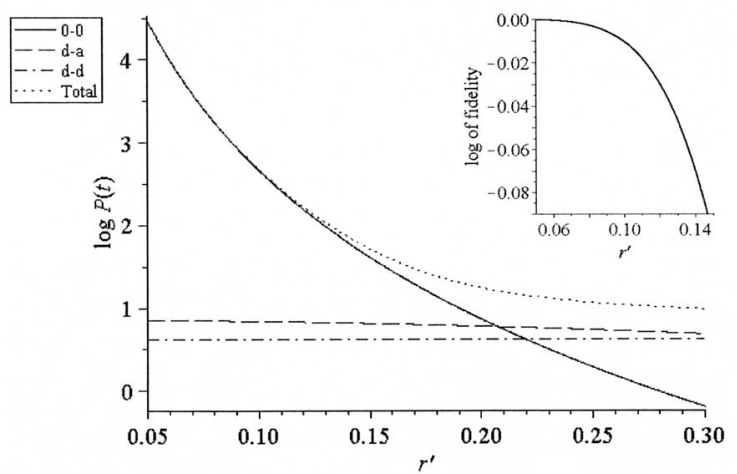

FIG. 13. Graph illustrating $\log P(t)$ against $r^{\prime}$ for pair of two dimensional hexagonal arrays. Here, the irradiance $I$ of the input laser is 1 $\times 10^{12} \mathrm{~W} \mathrm{~cm}^{-2}$.

much greater than 0.11 for an irradiance $I=1$ $\times 10^{12} \mathrm{~W} \mathrm{~cm}^{-2}$, or 0.025 for $1 \times 10^{10} \mathrm{~W} \mathrm{~cm}^{-2}$-values that are not particularly favorable in comparison to the previous two-dimensional system. This is explained by the fact that, in the case of the hexagonal lattice, the critical region close to the origin has a greater number of off-axis molecules permitting direct RET excitation.

\section{CONCLUSION}

The foregoing analysis has addressed necessary criteria and effective constraints surrounding the possible deployment of OCRET as a means of achieving all-optical switching. A number of issues flagged in earlier, preliminary work ${ }^{42-44}$ have now been resolved in detail. These include the necessity of utilizing molecules with (at least) two electronic excited levels in the appropriate uv/visible wavelength region-although it is reiterated that only off-resonant radiation is involved in the switching process. Several other facets have emerged from an identification of two specific symmetry classes, to which it is necessary that the donor and acceptor molecules belong. This latter constraint proves to be very readily satisfied as the two point groups concerned $-D_{2}$ and $C_{2 v}$-are well represented in common molecular forms; $C_{2 v}$ is especially prevalent, one family of examples being afforded by phenanthrene and its symmetric derivatives.

Moving onwards to considerations of device architecture and scale, the theory has then focused on three different

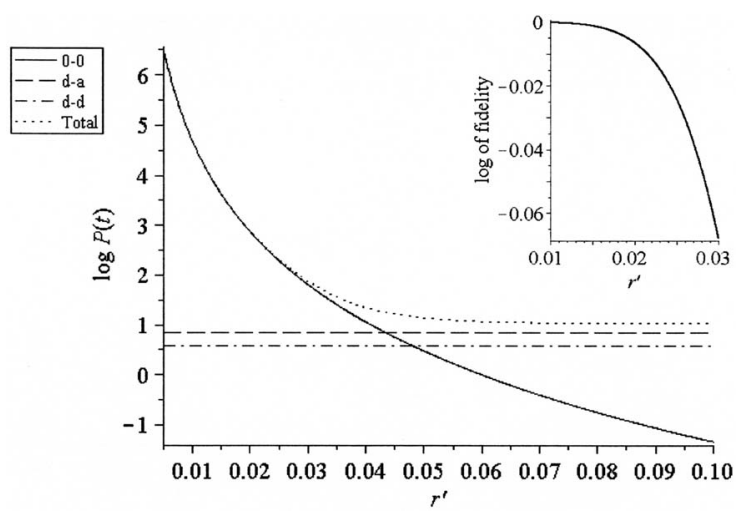

FIG. 14. Graph as Fig. 13, but for $I=1 \times 10^{10} \mathrm{~W} \mathrm{~cm}^{-2}$. 
types of array configuration. The concept of transfer fidelity, signifying the accuracy of mapping input to designated output, has been introduced and its key determinants have been identified from the form of the calculated results. Linear arrays prove to display the unusual property that their fidelity is independent of the throughput laser intensity, but in most other respects a pair of opposing square-planar arrays appears to offer the best prospects for implementation. The detailed analysis has shown that it is possible, by judicious choice of the relative values of the array spacing and lattice constant, together with the levels of laser intensity, to achieve arbitrarily low amounts of cross-talk. The laser pulse length will, in general, significantly exceed the time scale for the OCRET process and, therefore, it is reasonable to assume that the irradiance is effectively constant over time. It is worth noting that the OCRET contribution with a quadratic dependence on irradiance will be enhanced by focusing and pulse compression-the corresponding gains in efficiency more than compensating for diminished sample exposure.

Technical realizations of these model systems are likely to involve one of the considerable range of new nanofabrication methods. Generally, it may prove expedient to construct the donor and acceptor arrays as film layers, separated by a suitably transparent ultrathin spacer material. There is a possibility that this might indeed improve the energy transfer efficiency; this is a subject of ongoing investigation. Another aspect currently under exploration is the sensitivity to transition dipole disorder, i.e., the extent to which the orthogonality condition can be compromised before the OCRET mechanism loses its viability for all-optical switching. Organic dyes represent an extensive range of possibilities for choice of the donor and acceptor species; the use of quantum dots is also conceivable, though their relative isotropy could make it difficult to preclude conventional RET unless spinimprinted excitation and excitation transfer were to be engaged. ${ }^{61,62}$ Two techniques that might offer particular promise for the deposition and tailoring of the molecular components in each active layer are dip-pen nanolithography and thermochemical nanolithography, in each of which the potential to order and chemically modify molecular units has recently been established. ${ }^{63-66}$

In terms of applications, the achievement of optical switching in an extensive parallel-processing unit introduces a number of potential applications, beyond simple switching. Logic gate construction is an obvious possibility; the responsiveness to input modulation suggests other forms of action, possibly leading to optical transistor configurations. Our array results also signify that, for example, pixel-based images, written by donor excitation could be transferred with high fidelity to the acceptor film. In the realm of optical communications, possibilities might be built on the obvious capacity of such a system to act as an ultrafast information buffer; the high level of interest in such devices has already prompted others to explore "slow-light" methods, where a host of more problematic limitations apply. ${ }^{67,68}$ The systems we have presented offer numerous advantages: viable operation at short uv/visible wavelengths; the obviation of nonstandard, expensive optical elements; lack of any susceptibility to saturation problems; applicability to a host of molecular systems; a high information density optimized by using a single donoracceptor pair for each bit of information; ultrafast response with high repetition rate, high efficiency, and nanoscale miniaturization.

\section{ACKNOWLEDGMENTS}

We are grateful to the Leverhulme Trust for providing the financial support for this project.

${ }^{1}$ V. R. Almeida, C. A. Barrios, R. R. Panepucci, and M. Lipson, Nature (London) 431, 1081 (2004).

${ }^{2}$ T. Tanabe, M. Notomi, S. Mitsugi, A. Shinya, and E. Kuramochi, Opt. Lett. 30, 2575 (2005).

${ }^{3}$ T. Akiyama, N. Georgiev, T. Mozume, H. Yoshida, A. V. Gopal, and O. Wada, IEEE Photonics Technol. Lett. 14, 495 (2002).

${ }^{4}$ S. A. B. Nasrallaha, N. Sfina, and M. Said, Eur. Phys. J. B 47, 167 (2005).

${ }^{5}$ N. Iizuka, K. Kaneko, and N. Suzuki, Opt. Express 13, 3835 (2005).

${ }^{6}$ G. Sun, J. B. Khurgin, and R. A. Soref, Appl. Phys. Lett. 87, 201108 (2005)

${ }^{7}$ R. Akimoto, B. S. Li, K. Akita, and T. Hasama, Phys. Status Solidi B 243, 805 (2006).

${ }^{8}$ Y. Li, A. Bhattacharyya, C. Thomidis, T. D. Moustakas, and R. Paiella, Opt. Express 15, 5860 (2007).

${ }^{9}$ G. W. Cong, R. Akimoto, K. Akita, T. Hasama, and H. Ishikawa, Opt. Express 15, 12123 (2007).

${ }^{10}$ Y. Fedoryshyn, P. Strasser, P. Ma, F. Robin, and H. Jäckel, Opt. Lett. 32, 2680 (2007).

${ }^{11}$ Y. C. Chen, N. R. Raravikar, L. S. Schadler, P. M. Ajayan, Y. P. Zhao, T. M. Lu, G. C. Wang, and X. C. Zhang, Appl. Phys. Lett. 81, 975 (2002).

${ }^{12}$ H. Hippler, A. N. Unterreiner, J. P. Yang, S. Lebedkin, and M. M. Kappes, Phys. Chem. Chem. Phys. 6, 2387 (2004).

${ }^{13}$ B. S. Ham and P. R. Hemmer, Phys. Rev. Lett. 84, 4080 (2000).

${ }^{14}$ H. Schmidt and R. J. Ram, Appl. Phys. Lett. 76, 3173 (2000).

${ }^{15}$ P. Bermel, A. Rodriguez, S. G. Johnson, J. D. Joannopoulos, and M. Soljacic, Phys. Rev. A 74, 043818 (2006).

${ }^{16}$ A. W. Brown and M. Xiao, Opt. Lett. 30, 699 (2005).

${ }^{17}$ C. Y. Wang, Y. F. Chen, S. C. Lin, W. H. Lin, P. C. Kuan, and I. A. Yu, Opt. Lett. 31, 2350 (2006)

${ }^{18}$ J. P. Zhang, G. Hernandez, and Y. F. Zhu, Opt. Lett. 32, 1317 (2007).

${ }^{19}$ A. M. C. Dawes, L. Illing, S. M. Clark, and D. J. Gauthier, Science 308, 672 (2005).

${ }^{20}$ O. Boyraz, P. Koonath, V. Raghunathan, and B. Jalali, Opt. Express 12, 4094 (2004).

${ }^{21}$ H. Nakamura, Y. Sugimoto, K. Kanamoto, N. Ikeda, Y. Tanaka, Y. Nakamura, S. Ohkouchi, Y. Watanabe, K. Inoue, H. Ishikawa, and K. Asakawa, Opt. Express 12, 6606 (2004).

${ }^{22}$ A. Rostami, Microelectron. J. 37, 976 (2006).

${ }^{23}$ K. Asakawa, Y. Sugimoto, Y. Watanabe, N. Ozaki, A. Mizutani, Y. Takata, Y. Kitagawa, H. Ishikawa, N. Ikeda, K. Awazu, X. M. Wang, A. Watanabe, S. Nakamura, S. Ohkouchi, K. Inoue, M. Kristensen, O. Sigmund, P. I. Borel, and R. Baets, New J. Phys. 8, 208 (2006).

${ }^{24}$ Y. D. Wu, M. L. Huang, M. H. Chen, and R. Z. Tasy, Opt. Express 15, 9883 (2007).

${ }^{25}$ S. Diez, E. Hilliger, M. Kroh, C. Schmidt, C. Schubert, H. G. Weber, L. Occhi, L. Schares, G. Guekos, and L. K. Oxenloewe, Opt. Commun. 189, 241 (2001).

${ }^{26}$ H. J. S. Dorren, G. D. Khoe, and D. Lenstra, Opt. Commun. 205, 247 (2002).

${ }^{27}$ O. Wada, New J. Phys. 6, 183 (2004).

${ }^{28}$ M. F. Yanik, S. H. Fan, M. Soljacic, and J. D. Joannopoulos, Opt. Lett. 28, 2506 (2003).

${ }^{29}$ N. Moll, R. Harbers, R. F. Mahrt, and G. L. Bona, Appl. Phys. Lett. 88, 171104 (2006).

${ }^{30}$ M. R. Singh and R. H. Lipson, J. Phys. B 41, 015401 (2008).

${ }^{31}$ C. Janke, J. G. Rivas, P. H. Bolivar, and H. Kurz, Opt. Lett. 30, 2357 (2005).

${ }^{32}$ G. A. Wurtz, R. Pollard, and A. V. Zayats, Phys. Rev. Lett. 97, 057402 (2006).

${ }^{33}$ D. E. Chang, A. S. Sorensen, E. A. Demler, and M. D. Lukin, Nat. Phys. 3, 807 (2007).

${ }^{34}$ S. Z. Wu, F. Zeng, S. L. Yao, Z. Tong, W. L. She, and D. B. Luo, 
Macromolecules 36, 9292 (2003).

${ }^{35}$ Y. Luo, W. She, S. Wu, F. Zeng, and S. Yao, Appl. Phys. B: Lasers Opt. 80, 77 (2005).

${ }^{36}$ W. X. Que, X. Hu, X. L. Xia, and L. Zhao, Opt. Express 15, 480 (2007).

${ }^{37}$ P. Ormos, L. Fabian, L. Oroszi, E. K. Wolff, J. J. Ramsden, and A. Der, Appl. Phys. Lett. 80, 4060 (2002).

${ }^{38}$ P. F. Wu, D. Rao, B. R. Kimball, M. Nakashima, and B. S. DeCristofano, Appl. Phys. Lett. 81, 3888 (2002).

${ }^{39}$ Y. H. Huang, S. T. Wu, and Y. Y. Zhao, Opt. Express 12, 895 (2004).

${ }^{40}$ S. Roy, P. Sharma, A. K. Dharmadhikari, and D. Mathur, Opt. Commun. 237, 251 (2004).

${ }^{41}$ J. Topolancik and F. Vollmer, Appl. Phys. Lett. 89, 184103 (2006).

${ }^{42}$ D. L. Andrews and R. G. Crisp, J. Opt. A, Pure Appl. Opt. 8, S106 (2006).

${ }^{43}$ D. L. Andrews and R. G. Crisp, J. Fluoresc. 16, 191 (2006).

${ }^{44}$ R. G. Crisp and D. L. Andrews, Proc. SPIE 6195, 61951I (2006).

${ }^{45}$ Resonance Energy Transfer, edited by D. L. Andrews and A. A. Demidov (Wiley, Chichester, 1999).

${ }^{46}$ P. Allcock, R. D. Jenkins, and D. L. Andrews, Chem. Phys. Lett. 301, 228 (1999).

${ }^{47}$ P. Allcock, R. D. Jenkins, and D. L. Andrews, Phys. Rev. A 61, 023812 (2000).

${ }^{48}$ D. L. Andrews, R. G. Crisp, and S. Li, J. Chem. Phys. 127, 174702 (2007).

${ }^{49}$ D. L. Andrews and D. S. Bradshaw, Eur. J. Phys. 25, 845 (2004).

${ }^{50}$ D. L. Andrews, S. Naguleswaran, and G. E. Stedman, Phys. Rev. A 57, 4925 (1998).

${ }^{51}$ D. L. Andrews, L. C. D. Romero, and G. E. Stedman, Phys. Rev. A 67,
055801 (2003).

${ }^{52}$ P. W. Milonni and R. W. Boyd, Phys. Rev. A 69, 023814 (2004).

${ }^{53}$ R. Loudon and S. M. Barnett, J. Phys. B 39, S555 (2006).

${ }^{54}$ P. R. Berman, R. W. Boyd, and P. W. Milonni, Phys. Rev. A 74, 053816 (2006).

${ }^{55}$ S. Mukamel, Phys. Rev. A 76, 021803 (2007)

${ }^{56}$ I. Bialynicki-Birula and T. Sowinski, Phys. Rev. A 76, 062106 (2007).

${ }^{57}$ D. L. Andrews, L. C. Dávila Romero, and W. J. Meath, J. Phys. B 32, 1 (1999).

${ }^{58}$ E. Fermi, Nuclear Physics (University of Chicago Press, Chicago, 1950).

${ }^{59}$ D. L. Andrews and J. Rodriguez, J. Chem. Phys. 127, 084509 (2007).

${ }^{60}$ G. J. Daniels, R. D. Jenkins, D. S. Bradshaw, and D. L. Andrews, J. Chem. Phys. 119, 2264 (2003).

${ }^{61}$ B. W. Lovett, J. H. Reina, A. Nazir, and G. A. D. Briggs, Phys. Rev. B 68, 205319 (2003).

${ }^{62}$ G. D. Scholes and D. L. Andrews, Phys. Rev. B 72, 125331 (2005).

${ }^{63}$ R. D. Piner, J. Zhu, F. Xu, S. H. Hong, and C. A. Mirkin, Science 283, 661 (1999).

${ }^{64}$ C. A. Mirkin, S. H. Hong, and L. Demers, ChemPhysChem 2, 37 (2001).

${ }^{65}$ N. P. Reynolds, S. Janusz, M. Escalante-Marun, J. Timney, R. E. Ducker, J. D. Olsen, C. Otto, V. Subramaniam, G. J. Leggett, and C. N. Hunter, J. Am. Chem. Soc. 129, 14625 (2007).

${ }^{66}$ R. Szoszkiewicz, T. Okada, S. C. Jones, T. D. Li, W. P. King, S. R. Marder, and E. Riedo, Nano Lett. 7, 1064 (2007).

${ }^{67}$ R. W. Boyd and P. Narum, J. Mod. Opt. 54, 2403 (2007).

${ }^{68}$ K. L. Tsakmakidis, A. D. Boardman, and O. Hess, Nature (London) 450, 397 (2007) 